\title{
An Application of Convergence Acceleration Methods
}

\author{
George Fikioris
}

\begin{abstract}
It is pointed out that certain convergence acceleration methods can be applied to sequences of driving-point conductances obtained by solving Hallén's equation numerically. Five easily applied methods are considered and all seem to greatly improve convergence. Reasons for having confidence in the resulting extrapolations are discussed.
\end{abstract}

Index Terms - Dipole antennas, extrapolation, integral equations.

\section{INTRODUCTION}

W HEN solving an electromagnetics problem numerically, an important parameter is the number of basis functions (or grid cells). In practice, this parameter is frequently chosen based on the number of points per wavelength or other such a priori measures. For small problems, it is often feasible to make the parameter larger until the numerical solution is considered to have converged to a satisfactory final value. This process can yield greater accuracy; still, the associated "convergence" can be slow. For a relatively simple antenna problem, the present paper investigates the speed up of this process by contemporary extrapolation schemes.

Extrapolation generally means the estimation of a function at a point which is larger (or smaller) than all points at which the value of the function is known. Here, we are interested in estimations of the limit $s$ of a convergent sequence $\left(s_{N}\right)$ and, in particular, extrapolation schemes referred to as convergence acceleration methods (CAM's): Given a convergent sequence $\left(s_{N}\right)$ with unknown limit $s$, we seek a new sequence $\left(t_{N}\right)$ which converges faster to $s$ than $\left(s_{N}\right)$. More precisely [1], we require that the transformed sequence $\left(t_{N}\right)$ : (a) converges; (b) converges to the same limit $s$ as $\left(s_{N}\right)$ does; (c) that

$$
\lim _{N \rightarrow \infty} \frac{t_{N}-s}{s_{N}-s}=0 .
$$

A particular CAM is defined by a transformation rule, which can be linear or nonlinear. A well-known CAM [1] is the nonlinear Shanks transformation $e_{k}\left(s_{N}\right)(k=1,2, \cdots)$, usually implemented by means of the $\epsilon$ algorithm. For $k=1$, $e_{1}\left(s_{N}\right)$ is Aitken's $\Delta^{2}$ method. The subject of CAM's has many aspects. To start, for a given CAM one finds classes of

Manuscript received December 18, 1998; revised May 19, 1999. This work was done while the author was with the Air Force Research Laboratory, Sensors Directorate, Hanscom AFB, MA 01731-3010 and was supported by AFOSR under Project 2304IN01. Some of this work's final stages were supported by the Greek Secretariat of Research and Technology.

The author is with the Institute of Communication and Computer Systems, Department of Electrical and Computer Engineering, National Technical University of Athens, GR 157-73 Zografou, Athens, Greece.

Publisher Item Identifier S 0018-926X(99)09975-5. sequences for which (a)-(c) apply. A thorough discussion is provided in the comprehensive book [1] from which we have borrowed many special terms (some are in italics).

In numerical analysis, CAM's are used in numerical integration [1], [2], where $\left(s_{N}\right)$ is a sequence of numerically obtained estimates of an integral $s$, with $N$ related to the number of integrand evaluations. For example, $t_{N}$ can be formed by linearly combining $s_{N}$ and $s_{N-1}$ so that the leading terms in the large- $N$ expansions of the errors $s-s_{N}$ and $s-s_{N-1}$ are knocked out - this is the central idea in Romberg integration. Extrapolation here exploits the availability of asymptotic error estimates.

In electromagnetics, the idea of extrapolation is very common. Electromagnetic fields, for example, are frequently extrapolated to larger (or smaller) frequencies or spatial distances. In such cases, extrapolation schemes are often developed by utilizing the underlying physics. In his study of electromagnetic observations [3], Maxwell himself used what we now call the Shanks transformation $e_{2}\left(s_{N}\right)(k=2)$. More recently, CAM's have found extensive application in the calculation of certain Green's functions (see [4] and the references therein), and Sommerfeld integral tails [5]. In both these applications, the sequence elements $s_{N}$ are partial sums $\Sigma_{j}^{N} \alpha_{j}$ of infinite series. The later application, as well as CAM's in general, is discussed extensively in the recent review article [5]. There, choosing a CAM is facilitated by an investigation of the asymptotic behavior of the Sommerfeld integrand.

The specific purpose of this paper is to point out that certain CAM's, similar to those described in [5], can be applied to numerical solutions of the integral equation (2) below for the current $I(z)$ on a thin, tubular, cylindrical dipole, center-driven by a delta-function generator $V$. Our sequence elements $s_{N}=G_{N}$ are numerically obtained values of the driving-point conductance $s=G=\operatorname{Re}\{I(0) / V\}$ (the susceptance is infinite). These sequence elements result [6] by applying Galerkin's method with pulse functions (GMPF) to (2); the index $N$ corresponds to the number of pulses. The sequences $\left(G_{N}\right)$ thus obtained are monotone and, apparently, belong to the class of logarithmic sequences. These are often considered more challenging to accelerate. On the other hand, our sequences "look" very simple; simpler, for example, than those considered in [5], which oscillate.

As far as the author is aware, virtually nothing is known about convergence rates when GMPF is applied to (2). Indeed, some mathematical questions relevant to the integral equation (2) are very complicated, and have only been addressed 


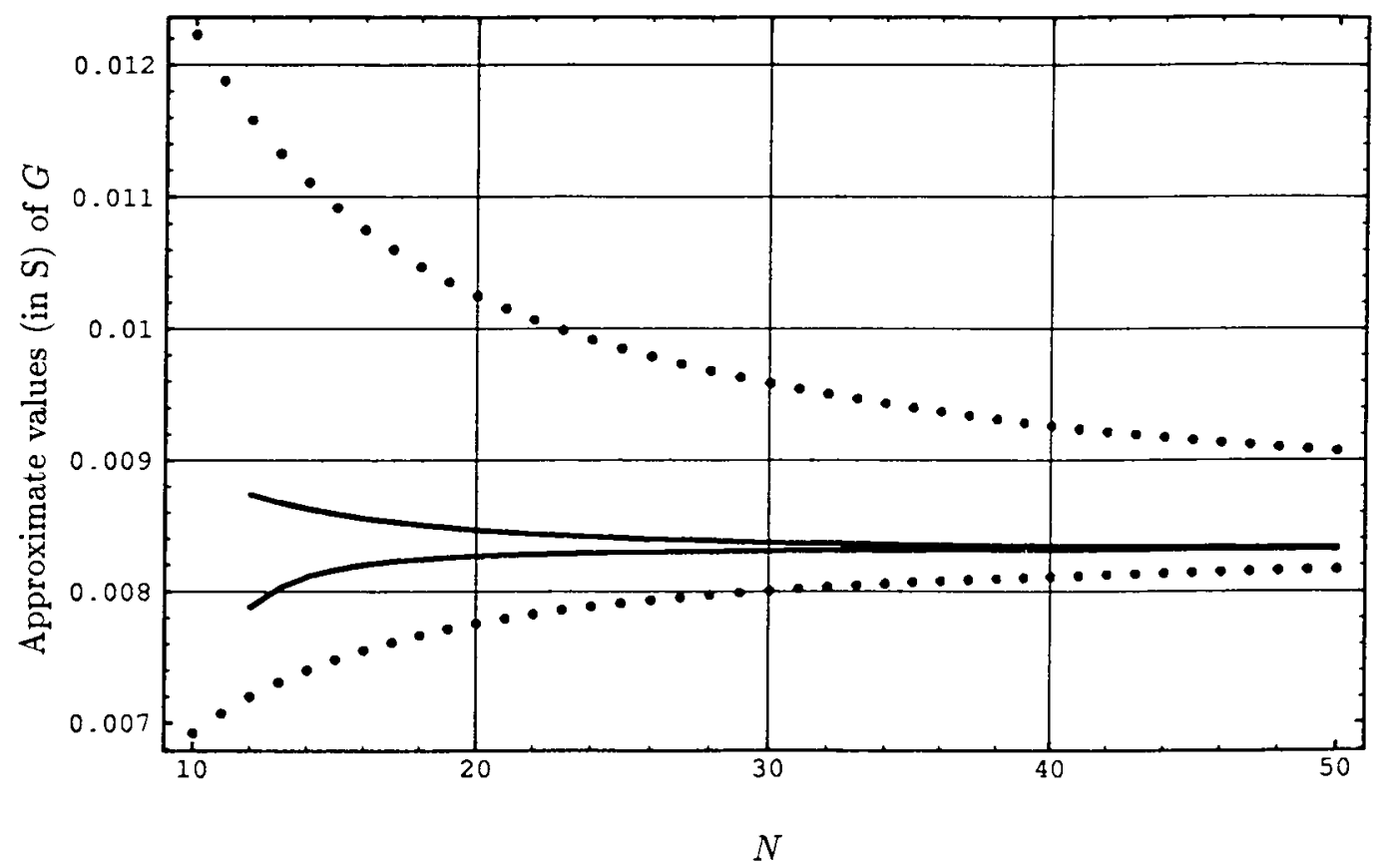

Fig. 1. $G_{N}^{\mathrm{a}}$ (dots-top) and $G_{N}^{\mathrm{b}}$ (dots-bottom) for $h / \lambda=0.25, a / \lambda=0.006$, and $N=10,11, \cdots, 50$; and corresponding sequence elements $T_{N}^{\mathrm{a}}$ (bottom solid line) and $T_{N}^{\mathrm{b}}$ (top solid line) resulting from the application of the CAM (5). When $N=50$ [i.e., $N=48$ in (5)], $T_{50}^{\mathrm{a}}=8.3208$ $\mathrm{mS}$, and $T_{50}^{\mathrm{b}}=8.3309 \mathrm{mS}$.

recently [7]. Therefore, no precise beforehand information is incorporated in our extrapolation schemes. However, we provide other reasons for having confidence in our methods. Although we do not attempt to come up with optimum methods, we indicate that certain popular CAM's, which are effective in other applications, are not well suited for the present problem.

\section{EXTRAPOLATION TECHNIQUES}

With $I( \pm h)=0$, Hallén's well-known integral equation under consideration is

$$
\begin{aligned}
\int_{-h}^{h} K_{\mathrm{ex}}\left(z-z^{\prime}\right) I\left(z^{\prime}\right) d z^{\prime} \\
\quad=\frac{i}{2 \zeta_{0}} V \sin \beta|z|+C \cos \beta z, \quad|z|<h \\
K_{\mathrm{ex}}(z)=\left(8 \pi^{2}\right)^{-1} \int_{-\pi}^{\pi} e^{i \beta R(z, \phi)} / R(z, \phi) d \phi \\
R(z, \phi)=\left[z^{2}+4 a^{2} \sin ^{2}(\phi / 2)\right]^{1 / 2}
\end{aligned}
$$

where $2 h$ and $a$ are the dipole's length and radius, $\beta=2 \pi / \lambda$ is the free-space wavenumber, and $\zeta_{0}=376.73 \Omega$. For $2 N+1$ pulse basis functions, each of width $z_{0}=2 h /(2 N+1)$, formulas [6] for the approximate value $G_{N}$ of driving-point conductance $G=\operatorname{Re}\{I(0) / V\}$, are listed in the Appendix. There are two alternative schemes (A.4a), (A.4b) for obtaining approximations $C_{N}$ to the constant $C$ and the corresponding $G_{N}$ 's will be denoted by $G_{N}^{\mathrm{a}}$ and $G_{N}^{\mathrm{b}}$. Both schemes result from $I(h)=0$; additionally, the square-root behavior of $I(z)$ near $z=h$ is incorporated in the second scheme, which is more precise. In what follows, we apply CAM's to $\left(G_{N}^{\mathbf{a}}\right)$ and $\left(G_{N}^{\mathrm{b}}\right)$ separately.
Representative plots of $G_{N}^{\mathrm{a}}$ and $G_{N}^{\mathrm{b}}(N=10,11, \cdots, 50)$ are shown in Fig. 1. As expected, both sequences appear to converge to the same limit, and $\left(G_{N}^{\mathrm{b}}\right)$ always appears to have a larger speed of convergence; but, even for $N=50$, the values $G_{N}^{\mathrm{a}}$ and $G_{N}^{\mathrm{b}}$ differ by $11 \%$. (This becomes $3 \%$ when $N$ is increased to 149). Each sequence somewhat resembles a "test" sequence of the form

$$
S_{N}=S+\gamma N^{-\mu}, \quad \mu>0
$$

which is a logarithmic sequence. Because there is a great number [1] of available CAM's, we use this rough similarity to narrow our search.

When applied to $\left(S_{N}\right)$, Aitken's $\Delta^{2}$ method yields a sequence $\left(P_{N}\right)$. We can show analytically that $P_{N}-S \sim$ $\gamma /(\mu+1) N^{-\mu}$ as $N \rightarrow \infty$ so that $\left(P_{N}\right)$ converges at the same rate as $\left(S_{N}\right)$. Thus, the $\Delta^{2}$ method (as well the iterated $\Delta^{2}$ method) does not seem adequate and we verified this by application to the actual $\left(G_{N}\right)$ 's. In other words, we have a contractive sequence transformation but no convergence acceleration. The well-known Richardson extrapolation [2] would require knowledge of $\mu$ so it is also not suitable.

Typically, transformed sequences are highly susceptible to noise in the original sequences [such as the noise arising from the numerical integration (A.2)] and to roundoff. This is especially true for "higher order" CAM's, which require many difference operations. In simple applications, however, we can usually detect such effects a posteriori.

The CAM's listed below resulted from examining [1] (and some of the references therein) in light of the aforementioned considerations. Most of these CAM's are "first-order" implementations of more general ones. The usual symbols $\Delta s_{N}=s_{N+1}-s_{N}$ and $\Delta^{2} s_{N}=\Delta s_{N+1}-\Delta s_{N}$ for difference 
operations are used

$$
\begin{aligned}
T_{N+2} & =G_{N+1}-2 \Delta G_{N} \Delta G_{N+1} / \Delta^{2} G_{N} \\
T_{N+3} & =G_{N+1}+\Delta G_{N+1} \Delta\left(1 / \Delta G_{N+1}\right) / \Delta^{2}\left(1 / \Delta G_{N}\right) \\
T_{N+1} & =\Delta\left(G_{N} / f_{N}\right) / \Delta\left(1 / f_{N}\right) ; \quad f_{N}=N \Delta G_{N-1} \\
T_{N+2} & =G_{N}-N\left(\Delta G_{N}\right)^{2} /\left[(N+1) \Delta G_{N+1}-N \Delta G_{N}\right] \\
T_{4 N} & =G_{4 N}-\left(G_{4 N}-G_{2 N}\right)^{2} /\left(G_{4 N}-2 G_{2 N}+G_{N}\right) .
\end{aligned}
$$

In each equation above we have chosen the index $k$ in $T_{k}$ according to the following helpful convention: $k$ coincides with the index of the most advanced term of $\left(G_{N}\right)$ necessary for the computation of $T_{k}$. Other choices for $k$ are possible and result in unimportant modifications. Apart from index changes and changes in algebraic form, (5)-(7) are first-order implementations of Wynn's [8] $\rho$-algorithm (5), Brezinski's [9] $\Theta$-algorithm (6), and what is usually called Levin's [10] $u$-transform (7). The CAM (6) is also called the Lubkin $W$-transformation. The CAM (8) is in [1, Theorem 3.7]. Finally, (9) results by applying the $\Delta^{2}$ method to subsequences $\left(G_{N}, G_{2 N}, \cdots\right)$ of $\left(G_{N}\right)$; in these, an original number $N$ in GMPF is successively doubled. For the test sequence $\left(S_{N}\right)$ of (4), it is easy to see analytically that (9) is superior to the normal $\Delta^{2}$ method. Note also that (9) utilizes terms of $\left(G_{N}\right)$ with small indexes.

When applied to $\left(G_{N}^{\mathrm{a}}\right)$ and $\left(G_{N}^{\mathrm{b}}\right)$, the CAM (5) gives the results seen in Fig. 1. The leftmost point in the solid line representing $\left(T_{N}^{\mathrm{a}}\right)$ [or $\left.\left(T_{N}^{\mathrm{b}}\right)\right]$ corresponds to $T_{12}^{\mathrm{a}}$ (or $T_{12}^{\mathrm{b}}$ ) and, in accordance with (5), was computed from the first three terms $G_{10}^{\mathrm{a}}, G_{11}^{\mathrm{a}}, G_{12}^{\mathrm{a}}$ (or $\left.G_{10}^{\mathrm{b}}, G_{11}^{\mathrm{b}}, G_{12}^{\mathrm{b}}\right)$; the rightmost point corresponds to $T_{50}^{\mathrm{a}}$ (or $T_{50}^{\mathrm{b}}$, respectively). In Fig. 1, the transformed sequences are smooth and seem free of noise/roundoff errors. The convergence appears to be accelerated substantially. Already when $N=12$, the values $T_{N}^{\mathrm{a}}, T_{N}^{\mathrm{b}}$ differ only by $10 \%$ and this rapidly decreases to $0.1 \%$ when $N=50$. The CAM's (6)-(9) also give very good results and numerical values which agree with those given by (5). For the specific parameters of Fig. 1, (5) seems to provide accurate answers somewhat faster than (6)-(9). A detailed comparison between (5)-(9), however, is beyond the scope of this paper.

Because we obtain the same answers by applying many CAM's to two independent sequences, we believe that our numerical results are correct and that all our CAM's are appropriate. We can also apply these CAM's to numerically obtained sequences approximating the imaginary part of $F\left(z_{1}\right)=I\left(z_{1}\right) / V-i 4 \beta a \zeta_{0}^{-1} \ln \beta z_{1}$, where $\beta z_{1}$ is small and fixed; such quantities [6] can be used to compute a drivingpoint susceptance. Finally, we expect these CAM's to be applicable to various scalar sequences arising from other integral/integrodifferential equations for the current distributions on wire antennas, including especially wire antennas of more complicated shapes.

\section{APPENDIX}

The value $G_{N}=\operatorname{Re}\left\{I_{N, 0} / V\right\}$ is obtained by applying GMPF to (2), as detailed in [6]: the approximate current $I_{N, 0}=I_{N, 0}^{(1)}+C_{N} I_{N, 0}^{(2)}$ at the driving point is found by first solving

$$
\begin{aligned}
& \sum_{n=-N}^{N} A_{l-n} I_{N, n}^{(1)}=B_{l}^{(1)} \\
& \sum_{n=-N}^{N} A_{l-n} I_{N, n}^{(2)}=B_{l}^{(2)}, \quad l=0, \pm 1, \cdots, \pm N
\end{aligned}
$$

where

$$
\begin{aligned}
A_{l} & =\int_{0}^{z_{0}}\left(z_{0}-z\right)\left[K\left(z+l z_{0}\right)+K\left(z-l z_{0}\right)\right] d z, \\
B_{l}^{(2)} & =\frac{2}{\beta} \sin \frac{\beta z_{0}}{2} \cos \left(\beta z_{0} l\right), \\
B_{l}^{(1)} & = \begin{cases}\frac{i 2 V}{\zeta_{0} \beta} \sin ^{2} \frac{\beta z_{0}}{4}, & \text { if } l=0 \\
\frac{i V}{\zeta_{0} \beta} \sin \frac{\beta z_{0}}{2} \sin \left(\beta z_{0}|l|\right), & \text { if } l \neq 0\end{cases}
\end{aligned}
$$

for $I_{N, n}^{(1)}$ and $I_{N, n}^{(2)}$ and then estimating $C$ from one of the alternative equations

$$
C_{N}=\left\{\begin{aligned}
& C_{N}^{\mathrm{a}}=-I_{N, N}^{(1)} / I_{N, N}^{(2)}, \quad \text { or } \\
& C_{N}^{\mathrm{b}}=-\left[\sqrt{3} I_{N, N}^{(1)}-I_{N, N-1}^{(1)}\right] / \\
& {\left[\sqrt{3} I_{N, N}^{(2)}-I_{N, N-1}^{(2)}\right] }
\end{aligned}\right.
$$

The choice $C_{N}=C_{N}^{\mathrm{a}}\left(C_{N}=C_{N}^{\mathrm{b}}\right)$ corresponds to the driving-point conductance $G_{N}^{\mathrm{a}}\left(G_{N}^{\mathrm{b}}\right)$.

\section{REFERENCES}

[1] C. Brezinski and M. Redivo Zaglia, Extrapolation Methods: Theory and Practice. New York: Elsevier, 1991.

[2] P. J. Davis and P. Rabinowitz, Methods of Numerical Integration, 2nd ed. Orlando, FL: Academic, 1984.

[3] J. C. Maxwell, A Treatise on Electricity and Magnetism, 3rd ed. Oxford: Clarendon, 1891. (New York: Dover, 1954, vol. 2, ch. XVI, reprint).

[4] N. Kinayman and M. I. Aksun, "Comparative study of acceleration techniques for integrals and series in electromagnetic problems," Radio Sci., vol. 30, no. 6, pp. 1713-1722, Nov./Dec. 1995.

[5] K. A. Michalski, "Extrapolation methods for Sommerfeld integral tails," IEEE Trans. Antennas Propagat., vol. 46, pp. 1405-1418, Oct. 1998.

[6] G. Fikioris and T. T. Wu, "On the application of numerical methods to Hallén's equation," IEEE Trans. Antennas Propagat., to be published.

[7] B. P. Rynne, "The well-posedness of the integral equations for thin wire antennas," IMA J. Appl. Math., vol. 49, pp. 35-44, 1992.

[8] P. Wynn, "On a procrustean technique for the numerical transformation of slowly convergent sequences and series," Proc. Cambridge Phil. Soc., vol. 52, pp. 663-671, 1956.

[9] C. Brezinski, "Accélération de suites à convergence logarithmique," $C$. R. Acad. Sci. Paris, vol. 273 A, pp. 727-730, 1971.

[10] D. Levin, "Development of nonlinear transformations for improving convergence of sequences," Int. J. Comput. Math., vol. 3, sec. B, pp. 371-388, 1973 .

George Fikioris, photograph and biography not available at the time of publication. 